\title{
Role of Choline in the Nutrition of the Rumen Protozoon Entodinium caudatum
}

\author{
By T. E. BROAD AND R. M. C. DAWSON \\ Biochemistry Department, Agricultural Research Council Institute of \\ Animal Physiology, Babraham, Cambridge CB2 $4 A T$
}

(Received I September I975)

\begin{abstract}
SUMMARY
A requirement of choline for the growth of Entodinium caudatum in a simplified culture medium has been demonstrated. Ethanolamine, $N$-methylethanolamine, or $N$-dimethylethanolamine were ineffective as substitutes. In the rumen, the normal environment of this organism, levels of free choline were virtually zero even after ingestion of pasture containing phosphatidylcholine which was rapidly catabolized. Free $\left[\mathrm{Me}^{14} \mathrm{C}\right]$ choline is very rapidly cleared from rumen fluid, a little being incorporated into the phosphatidylcholine of protozoa, but the clearance also occurs in animals with defaunated rumens. It is suggested that $E$. caudatum obtains choline for growth mainly from plant membrane material which it has ingested, rather than from the free base in the rumen liquor.
\end{abstract}

\section{INTRODUCTION}

Phosphatidylcholine is a major component of the phospholipids in the membranes of Entodinium caudatum (Dawson \& Kemp, 1967). Radioactive choline is rapidly taken up by the organism and incorporated into phosphatidylcholine through the cytidinediphosphatecholine pathway (Broad \& Dawson, 1975). In contrast to many other protozoa, phosphatidylcholine cannot be formed in $E$. caudatum by the $N$-methylation of phosphatidylethanolamine. Such fundamental biochemical observations suggested that choline might be an essential growth requirement for E. caudatum. By growing the organism in a simplified medium this requirement has now been demonstrated. In addition, observations have been made on the levels of free choline in the protozoon's normal environment, the rumen, and the clearance of choline from this organ.

\section{METHODS}

Growth of Entodinium caudatum. These cultures were established according to the procedure of Coleman (1962), with feeding on rice starch and dried grass every two days (Broad \& Dawson, 1975). The protozoa were then transferred to a simplified medium containing inorganic salts, sodium acetate, cysteine and chloramphenicol (Coleman, 1962) but without autoclaved sheep-rumen fluid. A suspension $(10 \%, \mathrm{w} / \mathrm{v})$ of $\beta$-sitosterol in the same basic medium was added. This was prepared by suspending $2 \mathrm{mg} \beta$-sitosterol (Sigma) in $0.1 \mathrm{ml}$ ethanol, adding $5 \mathrm{ml}$ of the simplified culture medium and sonicating in an ultrasonic water bath (Kerry's Ultrasonics Ltd, Basildon, Essex) for 2 min.

Estimation of choline. Choline in ethanolic extracts was acetylated to acetylcholine which was then assayed by its ability to contract frog rectus muscle (Eadie, Hebb \& Mann, I973). Ovine rumen fluid removed through a fistula was strained through muslin and mixed 
with 9 vol. of ethanol; this mixture was centrifuged and the supernatant used for choline assay. Good recoveries of choline standards added to rumen fluid were obtained. Rice starch was suspended in ethanol for $30 \mathrm{~min}$, and after centrifuging was extracted twice more with ethanol. Choline was estimated in the combined ethanol extracts.

Metabolism of leaf phosphatidylcholine in the rumen. Measurements were carried out as described by Dawson \& Hemington ( 974$) ;{ }^{14} \mathrm{C}$-labelled grass was prepared, administered to a sheep, and the rate of clearance of a particular component determined in relation to that of a non-metabolized component (carotene) of the same plant material. Phosphatidylcholine was separated and analysed as described below.

Clearance of choline and polyethylene glycol from the ovine rumen. Rumen-fistulated sheep were deprived of food overnight and $12.5 \mathrm{~g}$ polyethylene glycol (PEG 4000; Hopkin \& Williams) in $100 \mathrm{ml}$ water were introduced into the rumen. After $30 \mathrm{~min}, 200 \mathrm{ml}$ of rumen contents were removed, strained and 10 to $20 \mu \mathrm{Ci}\left[\mathrm{Me}^{-14} \mathrm{C}\right]$ choline (The Radiochemical Centre, Amersham, Buckinghamshire; $55 \mathrm{Ci} / \mathrm{mol}$ ) plus 2.5 to $25 \mathrm{mg}$ choline chloride were added. This mixture was then returned to the rumen. Serial samples of rumen contents $(20 \mathrm{ml})$ were taken over a 6 to $7 \mathrm{~h}$ period, strained through one layer of muslin to avoid undue loss of protozoa and centrifuged for $15 \mathrm{~min}$ at $21000 \mathrm{~g}$. A $3 \mathrm{ml}$ sample of the supernatant was assayed for polyethylene glycol (Hyden, 1955). The radioactivities present in further $0.5 \mathrm{ml}$ portions of the supernatant and precipitate were determined as described below.

Radioactivity determinations. Counts in $0.5 \mathrm{ml}$ portions of the rumen fluid supernatant were measured by liquid scintillation counting after mixing with $10 \mathrm{ml}$ Unisolve (KochLight). Because of appreciable quenching brought about by pigments in the rumen fluid, each sample was assayed with and without an internal standard of $\left[{ }^{4} \mathrm{C}\right]$ choline.

It proved difficult to assay bound $\left[{ }^{14} \mathrm{C}\right]$ choline directly in the rumen fluid precipitate. Since phosphatidylcholine and lysophosphatidylcholine are the only choline-containing phospholipids in rumen micro-organisms, the following procedure was adopted. The precipitate from $20 \mathrm{ml}$ rumen fluid was well mixed with $7 \mathrm{ml}$ methanol, and $14 \mathrm{ml}$ chloroform was added. After thorough shaking and standing for $15 \mathrm{~min}, 4 \mathrm{ml}$ water was added. The mixture was centrifuged and the lower phase collected and washed with an equal volume of chloroform-methanol- $0.9 \% \mathrm{NaCl}(3: 47: 49$, by vol.). The volume of the lower phase was then measured. The phospholipids in a $2 \mathrm{ml}$ sample were degraded with alkali at $37^{\circ} \mathrm{C}$. After neutralization with ethyl formate (Dawson, Hemington \& Davenport, I962), the $\left[{ }^{14} \mathrm{C}\right] \mathrm{glyceryl}$ hosphorylcholine was cleanly separated from plant pigments using the two-phase solvent system described; the pigments remained in the less polar phase. A sample $(0.5 \mathrm{ml})$ of the upper phase was mixed with $10 \mathrm{ml}$ Unisolve scintillation fluid and counted.

Reproducibility. All the experiments reported in this paper were repeated at least three times, with essentially similar results. Each curve in Figs. I to 4 represents one typical experiment.

\section{RESULTS}

\section{Culture of E. caudatum in simplified medium}

Entodinium caudatum could be grown in a simplified medium consisting of inorganic salts, sodium acetate, cysteine and chloramphenicol (Coleman, I962) providing $\beta$-sitosterol was present. Plant sterols are an essential requirement for E. caudatum and substitute effectively for the dried grass and rumen fluid also normally thought to be essential for growth (Dawson and Broad, unpublished observations). When grown on this simplified 


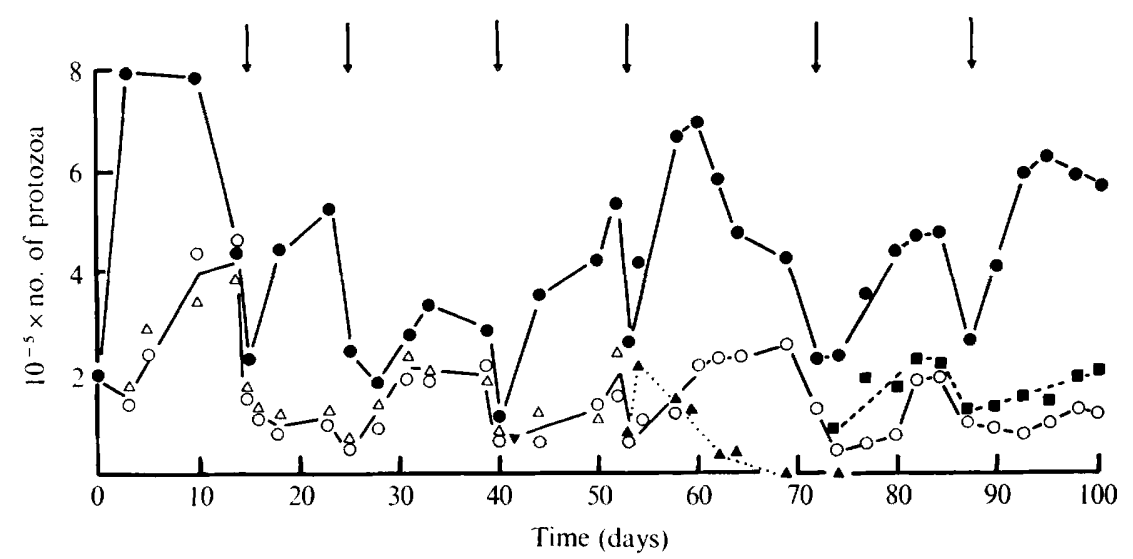

Fig. I. Growth of E. caudatum in full and simplified culture media and the effect of choline lack and supplementation. The control culture (O) was grown under Coleman's ( 1962 ) recommended conditions with dried grass and starch feeding. Simplified medium containing $\beta$-sitosterol and feeding with impure rice starch (containing choline) were used for two further cultures $(\triangle, O)$. At 53 days one of these $(\therefore)$ was fed instead on purified rice starch without choline $(\Delta)$. At 72 days the other culture (C) was split, with one part (O) continuing as before while the other $(\boldsymbol{\square})$ was fed pure rice starch supplemented with choline (10 $\mu \mathrm{g} / 50 \mathrm{ml}$ culture medium). The points at which the cultures were divided are indicated by arrows.

medium, with rice starch feeding every two days, cultures remained viable and dividing for up to 386 days.

\section{Requirements for choline}

Since E. caudatum is incapable of synthesizing choline (Broad \& Dawson, I975), as are the associated bacteria, the successful maintenance of cultures without added choline was unexpected (Fig. 1). It was anticipated that choline would be required for the formation of new membrane material during growth and division since phosphatidylcholine is a major component of these membranes (Dawson \& Kemp, 1967). As E. caudatum cells obtained from such cultures still contained phosphatidylcholine, it was possible that the cultures were obtaining choline from an unsuspected source. Analysis of the commercial rice starch (BDH) revealed that it contained $13 / \mathrm{g}$ free choline/g, although no phosphatidylcholine could be detected. Purification of the rice starch (Whelan, 1955) reduced the choline content to less than $0 . \mathrm{I} \mu \mathrm{g}$ choline $/ \mathrm{g}$; when this purified rice starch was fed to cultures of E. caudatum in simplified medium they died within ten days. However, if the purified starch was supplemented with $10 \mu \mathrm{g}$ choline per feed (30 mg rice starch $/ 50 \mathrm{ml}$ culture) then the protozoa grew as satisfactorily as those fed non-purified rice starch (Fig. 1). Replicate experiments confirmed that choline-supplemented medium could keep $E$. caudatum alive and active for up to 125 days (the maximum tested); cultures died immediately the choline was withdrawn. When possible precursors of choline such as ethanolamine, $N$-monomethylethanolamine and $N$-dimethylethanolamine were added at the same concentration they were ineffective and cultures died in much the same time as when choline had been omitted.

\section{Choline levels in ovine rumen fluid}

The normal environment of E. caudatum, i.e. ovine rumen liquor, was analysed to determine the level of free choline. Virtually no choline could be detected in the fasting animal, and choline $(<1 \mathrm{nmol} / \mathrm{ml})$ appeared only transiently after the animal had eaten $600 \mathrm{~g}$ grass (Table I). Complete recovery of the choline added to rumen fluid at low levels was 
Table I. Free choline content of ovine rumen fluid supernatant during feeding and starvation

Animals were starved for $24 \mathrm{~h}$ (Expt 1 ) or $17 \mathrm{~h}($ Expt 2), then fed $600 \mathrm{~g}$ grass between 10.30 and I I.30 (Expt I) or IO.I 5 and I I.30 (Expt 2), followed by further starvation.

$\begin{array}{lccc}\text { Time } & \begin{array}{c}\text { Choline level } \\ (\mathrm{nmol} / \mathrm{ml})\end{array} & \text { Time } & \begin{array}{c}\text { Choline level } \\ (\mathrm{nmol} / \mathrm{ml})\end{array} \\ 10.30 & 0.5 & 09.45 & <0.2 \\ 11.30 & 0.8 & 10.15 & 0.4 \\ 12.00 & 0.7 & 11.00 & 0.6 \\ 13.00 & <0.2 & 12.00 & <0.2 \\ 14.30 & <0.2 & 13.00 & <0.2 \\ 17.00 & <0.2 & 14.30 & <0.2 \\ 09.30 & <0.2 & 17.00 & <0.2 \\ & & 09.30 & <0.2\end{array}$

obtained. The grass fed would contain about 800 to $900 \mu$ mol phosphatidylcholine, which if completely broken down should have liberated approximately $\mathrm{I} 60 \mathrm{nmol}$ choline $/ \mathrm{ml}$ rumen fluid. That phosphatidylcholine breakdown occurs was confirmed in the experiments described in the next section.

\section{Breakdown of leaf phosphatidylcholine by rumen contents}

Although experiments with pure ${ }^{32}$ P-labelled phosphatidylcholine have shown a breakdown of this phospholipid by rumen micro-organisms (Dawson, 1959), it was not known whether this was true for leaf phosphatidylcholine complexed in the insoluble lipoproteins of plant membranes. Consequently, two types of experiments were undertaken.

(i) A sheep was given $450 \mathrm{~g}$ of grass uniformly labelled with ${ }^{14} \mathrm{C}$, and serial samples were withdrawn through a fistula in the rumen. The radioactivity of the phosphatidylcholine isolated from a given volume of rumen contents was compared with the activity of $\beta$ carotene obtained from the same volume. $\beta$-Carotene is not metabolized in the rumen (Dawson \& Hemington, 1974) and thus the comparison indicated the rate of decomposition of the grass phosphatidylcholine, irrespective of the rate of rumen clearance or the amount of radioactivity in the sample of rumen contents withdrawn. A very rapid decomposition of the plant phosphatidylcholine had occurred (Fig. 2).

(ii) Pasture grass was homogenized with sheep rumen contents ( $\mathrm{I} / 10 \mathrm{ml}$ ) and incubated under $\mathrm{N}_{2}-\mathrm{CO}_{2}$ (I9:I, v/v) at $38{ }^{\circ} \mathrm{C}$. Lipids were extracted from serial samples and the phospholipids separated and estimated by thin-layer chromatography. After 30, 60 and I $20 \mathrm{~min}, 28,23$ and II \% respectively, of the original phosphatidylcholine in the system remained (Fig. 2). No evidence for the accumulation of lysophosphatidylcholine was obtained.

\section{Clearance of free choline from the ovine rumen}

Because of the extremely low levels of free choline present in the rumen even in animals digesting fodder containing large amounts of choline precursors, the rate of clearance of free choline from the organ was tested. This rate was extremely rapid; after administering $25 \mathrm{mg}\left[\mathrm{Me}^{-14} \mathrm{C}\right] \mathrm{choline}$ chloride through a rumen fistula to a sheep starved overnight, $50 \%$ of the activity had disappeared within $90 \mathrm{~min}$ (Fig. 3), while with a $2.5 \mathrm{mg}$ dose little choline remained after $\mathrm{I} h$. Probably most of the radioactivity remaining after several hours 


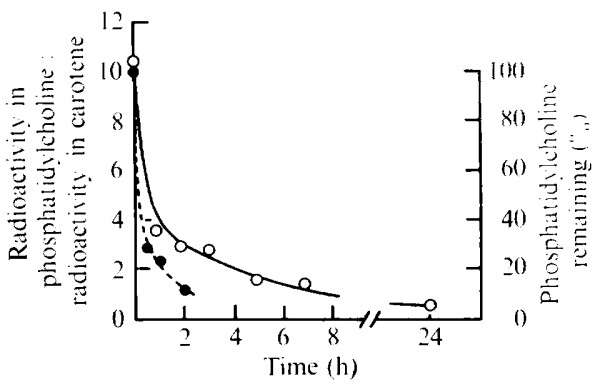

Fig. 2

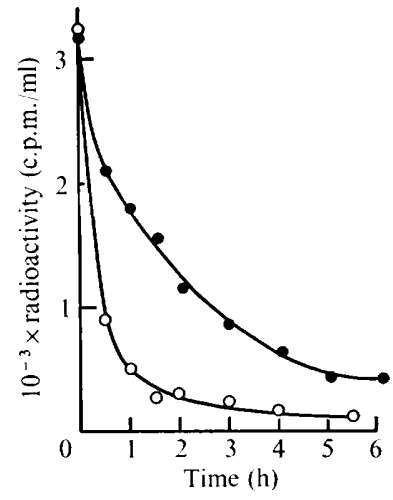

Fig. 3

Fig. 2. Digestion of grass leaf phosphatidylcholine by ovine rumen contents in vivo and in vitro. In the in vivo experiment a sheep was given $450 \mathrm{~g}$ grass labelled with ${ }^{14} \mathrm{C}$, serial samples of rumen fluid were withdrawn through a fistula and the ratio of radioactivity in phosphatidylcholine and $\beta$-carotene was determined $(0)$. In the in vitro experiment rumen contents were withdrawn and homogenized (Ultraturrax) with $10 \%(\mathrm{w} / \mathrm{v})$ grass. The percentage disappearance of phosphatidylcholine from the homogenate during anaerobic incubation at $38^{\circ} \mathrm{C}$ was determined

Fig. 3. Disappearance of radioactivity from rumen contents' supernatant fraction after administration of $\left[\mathrm{Me}-{ }^{14} \mathrm{C}\right]$ choline. $\left[\mathrm{Me}-{ }^{14} \mathrm{C}\right]$ choline $(10 \mu \mathrm{Ci})$ was administered through a rumen fistula together with $25 \mathrm{mg}(\mathbf{)})$ or $2.5 \mathrm{mg}(0)$ choline chloride. Serial samples of rumen contents were withdrawn and after centrifugation the radioactivity was measured in each portion. The level at zero time was computed from the dose administered and the rumen volume measured with polyethylene glycol.
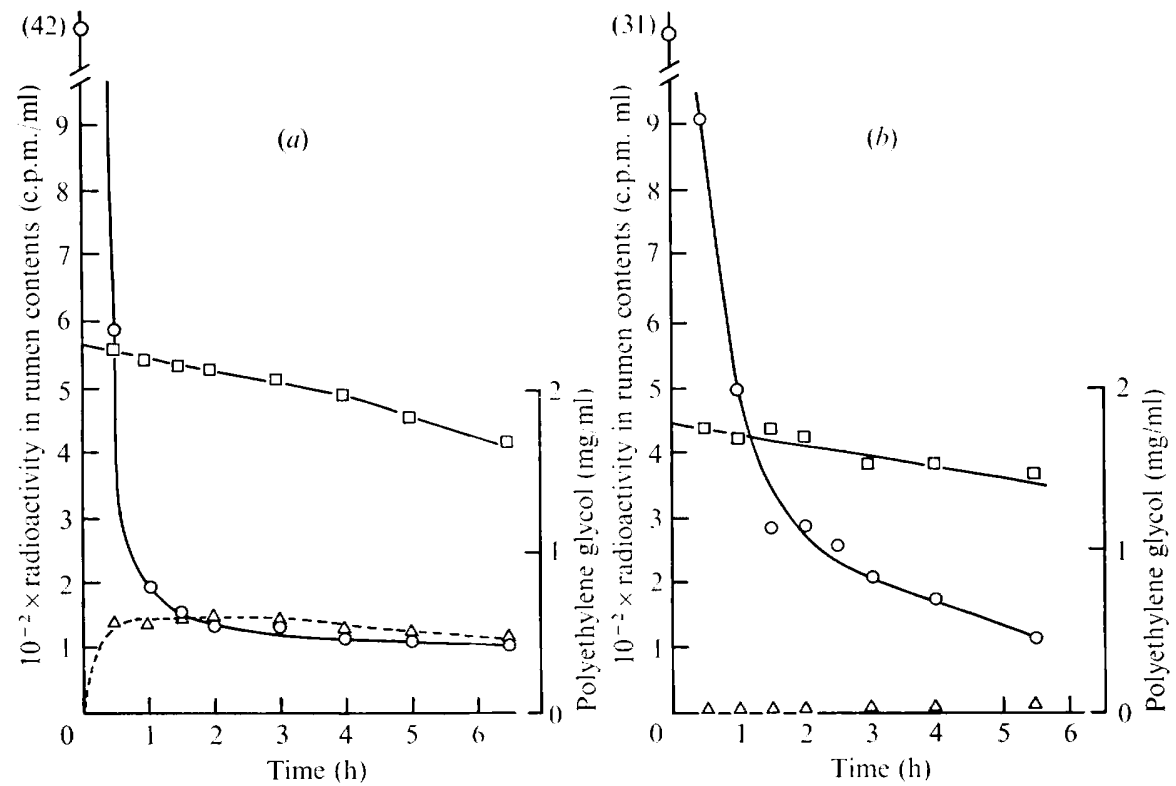

Fig. 4. Disappearance of radioactivity of administered $\left[M e-{ }^{14} \mathrm{C}\right] \mathrm{choline}$ from normal and defaunated rumens of sheep and the appearance of label in phosphatidylcholine. $\left[M e-{ }^{14} \mathrm{C}\right]$ choline $(2.5 \mathrm{mg})$ and of polyethylene glycol $(12.5 \mathrm{~g})$ were administered through a rumen fistula. Disappearance of radioactivity from the supernatant fraction (1)) and appearance of radioactivity in the phosphatidylcholine $(\triangle)$ are shown in a normal rumen $(a)$ and a defaunated rumen $(b)$. The level of polyethylene glycol $(\square)$ gives a measure of rumen emptying. 
represents a metabolite of choline which is not rapidly cleared from the rumen. Thus direct choline analysis of the rumen supernatant indicated a level far lower than that computed from the specific radioactivity of the administered dose. In addition, the 'stable' radioactivity could be separated from choline by paper ionophoresis. The choline clearance could not be accounted for by rumen emptying, since polyethylene glycol administered at the same time showed little loss compared with choline and there was little incorporation of $\left[{ }^{14} \mathrm{C}\right]$ choline into protozoal phosphatidylcholine (Fig. 4). Furthermore, free choline was still lost from the rumen of a sheep from which ciliated protozoa had previously been eliminated by detergent treatment (Dawson \& Kemp, 1969) and which consequently was devoid of all ability to synthesize phosphatidylcholine from $\left[{ }^{14} \mathrm{C}\right]$ choline (Fig. 4). In addition, virtually no loss of water-soluble radioactivity occurred when radioactive choline at a concentration equivalent to that in the in vivo experiments was incubated anaerobically with withdrawn rumen contents.

\section{DISCUSSION}

The prediction of a choline requirement for the nutrition of E. caudatum, based on fundamental compositional and metabolic studies on the organism (Dawson \& Kemp, 1967; Broad \& Dawson, 1975), has been confirmed. The fact that ethanolamine or its $\mathrm{N}$-monomethyl or $\mathrm{N}$-dimethyl derivatives could not subsitute for this requirement is perhaps not surprising since the organism seems incapable of the required type of methylation reactions for the synthesis of choline from these precursors (Broad \& Dawson, 1975).

Presumably, for the continued proliferation of this organism in the rumen it must synthesize phosphatidylcholine, a major component of its cell membranes. The free choline level in the rumen was extremely low, even when the sheep had recently eaten foodstuffs containing choline precursors. The usual level of free choline available would certainly not be able to support growth of the protozoon in vitro, even though the organism possesses an extraordinarily rapid rate of choline uptake (Broad \& Dawson, I975) which would help to accumulate the base when it appeared transiently during ruminal digestion. Unless any choline taken up could be stored and used up gradually for the necessary membrane synthesis in cell division, the growth of E. caudatum in the intact rumen could be expected to be intermittent. Perhaps a more probable explanation is that the organism takes into its vacuoles plant particulate matter (Hall, West \& Coleman, 1974) which is much more gradually broken down than that in the rumen liquor itself, thus supplying the necessary choline over a much longer period.

Leaf phosphatidylcholine is rapidly broken down by rumen contents both in vitro and in vivo. The capacity for carrying out this breakdown in vitro is substantially destroyed by boiling the rumen contents, so it is more likely that the enzymes involved are largely microbial rather than that they arise from the leaf tissue itself; rumen bacteria with active phospholipases have recently been isolated (Hazlewood \& Dawson, 1975). Since lysophosphatidylcholine is not observed to accumulate and many rumen micro-organisms contain an active glycerylphosphorylcholine diesterase (Hazlewood \& Dawson, 1975), it is to be expected that the destruction of leaf phosphatidylcholine would be associated with the liberation of free choline. Additionally, choline would enter the rumen through the saliva during feeding (our estimations on bovine saliva have indicated a level of Io nmol choline $/ \mathrm{ml}$ ).

That free choline does not accumulate in significant amounts during, and after, feeding is undoubtedly due to the extremely rapid clearance of this base from the rumen, as revealed by our experiments with radioactive choline. Synthesis of protozoal phospholipids or rumen 
emptying could account for the choline disappearance to only a very limited extent. Since the loss does not occur in isolated rumen contents, the implication is that there is very rapid absorption through the rumen wall. If this is true then the rumen epithelial cells must possess powerful choline pumps, since preliminary measurements have shown that plasma choline levels in the sheep are appreciably above those in the rumen.

T. E. B. was supported by a Sir Walter Mulholland fellowship from the New Zealand Meat Board. We are grateful to Dr G. S. Coleman for providing the initial E. caudatum cultures. Mr S. Mann is thanked for his expert analysis of the free choline content of ovine and bovine body fluids.

\section{REFERENCES}

Broad, T. E. \& Dawson, R. M. C. (I975). Phospholipid biosynthesis in the anaerobic protozoon Entodinium caudatum. Biochemical Journal 146, 317-328.

Coleman, G. S. (1962). The preparation and survival of almost bacteria-free suspensions of Entodinium caudatum. Journal of General Microbiology 28, 27I-28I.

Dawson, R. M. C. (1959). Hydrolysis of lecithin and lysolecithin by rumen micro-organisms of the sheep. Nature, London 183 , I $822-1823$.

Dawson, R. M. C. \& Hemington, N. (I974). Digestion of grass lipids and pigments in the sheep rumen. British Journal of Nutrition 32, 327-340.

Dawson, R. M. C., Hemington, N. \& Davenport, J. B. (1962). Improvements in the method of determining individual phospholipids in a complex mixture by successive chemical hydrolyses. Biochemical Journal 84, 497-50 I.

Dawson, R. M. C. \& KEMP, P. (1967). The aminoethylphosphonate-containing lipids of rumen protozoa. Biochemical Journal 105, $837-842$.

Dawson, R. M. C. \& Kemp, P. (I969). The effect of defaunation on the phospholipids and on the hydrogenation of unsaturated fatty acids in the rumen. Biochemical Journal $\mathbf{1} 5 \mathbf{5}, 35 \mathrm{I}-352$.

EAdie, I., HebB, C. \& MANN, S. P. (1973). Free choline levels in the rat brain. Journal of Neurochemistry 20 , I $499-1502$.

Hall, F. J., West, J. \& Coleman, G. S. (1974). Fine structural studies on the digestion of chloroplasts in the rumen ciliate Entodinium caudatum. Tissue and Cell 6, 243-253.

Hazlewood, G. P. \& Dawson, R. M. C. (1975). Isolation and properties of a phospholipid-hydrolysing bacterium from ovine rumen fluid. Journal of General Microbiology 89, I63-174.

HYISEN, S. ( 1955). A turbidimetric method for the determination of higher polyethylene glycols in biological materials. Lantbrukshögskolans Annaler 22, 139-145.

Whelan, W. J. ( I955). Starch, glycogen, fructosans and similar polysaccharides. In Modern Methods of Plant Analysis, vol. 2, pp. I45-196. Edited by K. Paech and M. V. Tracey. Berlin: Springer-Verlag. 